\title{
Tools for road infrastructure safety management in Poland
}

\author{
Wojciech Kustra ${ }^{1, *}$, and Lech Michalski ${ }^{1}$ \\ ${ }^{1}$ Gdansk University of Technology, Faculty of Civil and Environmental Engineering, Gdansk, Poland
}

\begin{abstract}
Road safety can be improved by implementing principles of road safety infrastructure management (RIS) on the network of European roads as adopted in the Directive. The document recommends that member states should use tried and tested tools for road safety management such as: road safety impact assessment (RIA), road safety audit (RSA), safety management on existing road networks including road safety ranking (RSM) and road safety inspection (RSI). The objective of the methods is to help road authorities to take rational decisions in the area of road safety and road infrastructure safety and understand the consequences occurring in the particular phases of road life cycle. To help with assessing the impact of a road project on the safety of related roads, a method was developed for longterm forecasts of accidents and accident cost estimation as well as a risk classification to identify risks that are not acceptable risks. With regard to road safety audits and road safety inspection, a set of principles was developed to identify risks and the basic classification of mistakes and omissions.
\end{abstract}

\section{Introduction}

Poland continues to be one of the European Union's worst performing countries for road deaths. In 2015 there were 2,938 people killed on Polish roads with 39,800 people injured. While the priorities set out in national and regional road safety programmes [1] help to systematically reduce Poland's road deaths, the results are far from what is expected. Road safety can be improved by implementing principles of road safety infrastructure management (RIS) on the network of European roads as adopted in the Directive [2]. RIS management involves the use of procedures throughout the life cycle of a road. The purpose of the procedures is to identify road hazards systematically, assess the possible consequences for road users, use measures to eliminate the hazards or mitigate the consequences. The consequences are measured with the number of accidents, injured and killed in road accidents and the costs of road accidents. The document recommends that member states should use tried and tested tools for road safety management such as:

- road safety impact assessment (RIA),

- road safety audit (RSA),

- safety management on existing road networks:

o road safety ranking (RSM),

$\circ$ road safety inspection $(\mathrm{RSI})$.

* Corresponding author: castrol@.pg.gda.pl 
To meet the needs of road authorities (national, regional and local), the Gdansk University of Technology (Department of Highway Engineering) in cooperation with the Krakow University of Technology (Department of Road Construction and Road Traffic Engineering) have developed several basic tools for managing the safety of Poland's road infrastructure [3-5]. Tools for managing safety on road networks have been developed by a team at the University of Science and Technology in Bydgoszcz [6].

\section{Methodology basis}

The purpose of the method is to help road authorities to take rational decisions about road safety, road infrastructure safety and the consequences that occur in the various stages of the life cycle of a road structure [1]. Work on building the particular elements of Poland's road infrastructure safety management adopted the following assumptions:

- the management system and its elements will cover all stages of a road structure's life cycle (planning, design, construction, operation and closure),

- road safety infrastructure management is based on risk management,

- a variety of methods to identify hazards and sources of hazards will be used.

A risk management method is a repetitive procedure designed to reduce road traffic risk effectively and efficiently with particular emphasis on interventions and measures related to road infrastructure within reasonable limits. The proposed risk management method includes two distinctive phases:

- risk assessment phase, process of analysing and determining acceptable risk taking into account standards for risk acceptance,

- risk response phase with three important phases: handling risk, monitoring risk and communicating risk.

The practice of road safety improvement just as in medicine, uses two types of therapy: treating the symptoms or treating the causes.

- Treating the symptoms means to identify the risk of hazards and their sources by analysing past road accidents and their causes.

- Treating the causes means to identify hazards and sources of hazards by conducting inspections in the field (on an existing road) or auditing designs (a planned road or one being designed) and by analysing expected (forecasted) road accidents and their consequences.

\section{Method of impact assessment}

Assessing the impact of a planned road on road safety involves a strategic analysis of how the variants of a road will affect road safety on a network of public roads within the planned road's impact area [7]. The Road Impact Assessment (RIA) is conducted to rank the variants of the planned road by their impact on road safety within the network of roads that are within the planned road's impact zone. The results of the analysis should be included in a multicriteria analysis (together with other criteria: technical, economic and environmental criteria) when assessing the variants of the road under analysis. The road safety impact assessment should also be used to reject from further design stages those variants that do not meet basic road safety standards. The research problem was to develop a method for forecasting road safety measures such as accident density $\mathrm{AD}$, injury density ID and killed density KD also known as measures of societal risk. Measures of societal risk are calculated using the following relations (example for accident density AD) (1):

$$
A D_{i, j, v, k}=\beta_{1, A, 1} \cdot Q_{i, j, v}^{\beta_{2, A, k}} \cdot \exp \left(\beta_{3, A, k} \cdot Q_{i, j, v}+\beta_{4, A, k} \cdot P H V_{i, j, v}\right) \cdot f_{T P} \cdot f_{R L} \cdot f_{A E} \cdot f_{D I}
$$


where:

- $Q_{i, j, v}$ - average annual daily traffic on the analysed road section $\mathrm{j}$, for the year of the forecast i, variant v (thou. veh./ 24h),

- $P H V_{i, j, v}$ - share of heavy vehicles (trucks and buses) on the analysed road section $\mathrm{j}$ in forecast year $\mathrm{i}$, for variant $\mathrm{v}(\%)$,

$-\beta_{1}, \ldots \beta_{n}$, - equation coefficients,

- $k$ - number of carriageways, $\mathrm{k}=1$ one carriageway, $\mathrm{k}=2$ two carriageways,

- $f_{T P}$ - rate of the effect of the year of forecast which takes account of the level of socioeconomic development of a country and systemic actions designed to improve road safety, - $\boldsymbol{f}_{\boldsymbol{R} L}$ - rate of the effect of road location (curvature, waviness, region of the country),

- $\boldsymbol{f}_{A E}$ - rate of the effect of the type of roadside (urban, industrial, rural, wooded) cut across by the analysed road section,

- $\boldsymbol{f}_{\boldsymbol{D} I}-$ rate of the effect of junction or interchange density DI.

Fig. 1 shows selected road safety measures depending on ADDT intensity. The values for road class $\mathrm{A}$ and $\mathrm{S}$ are clearly lower.
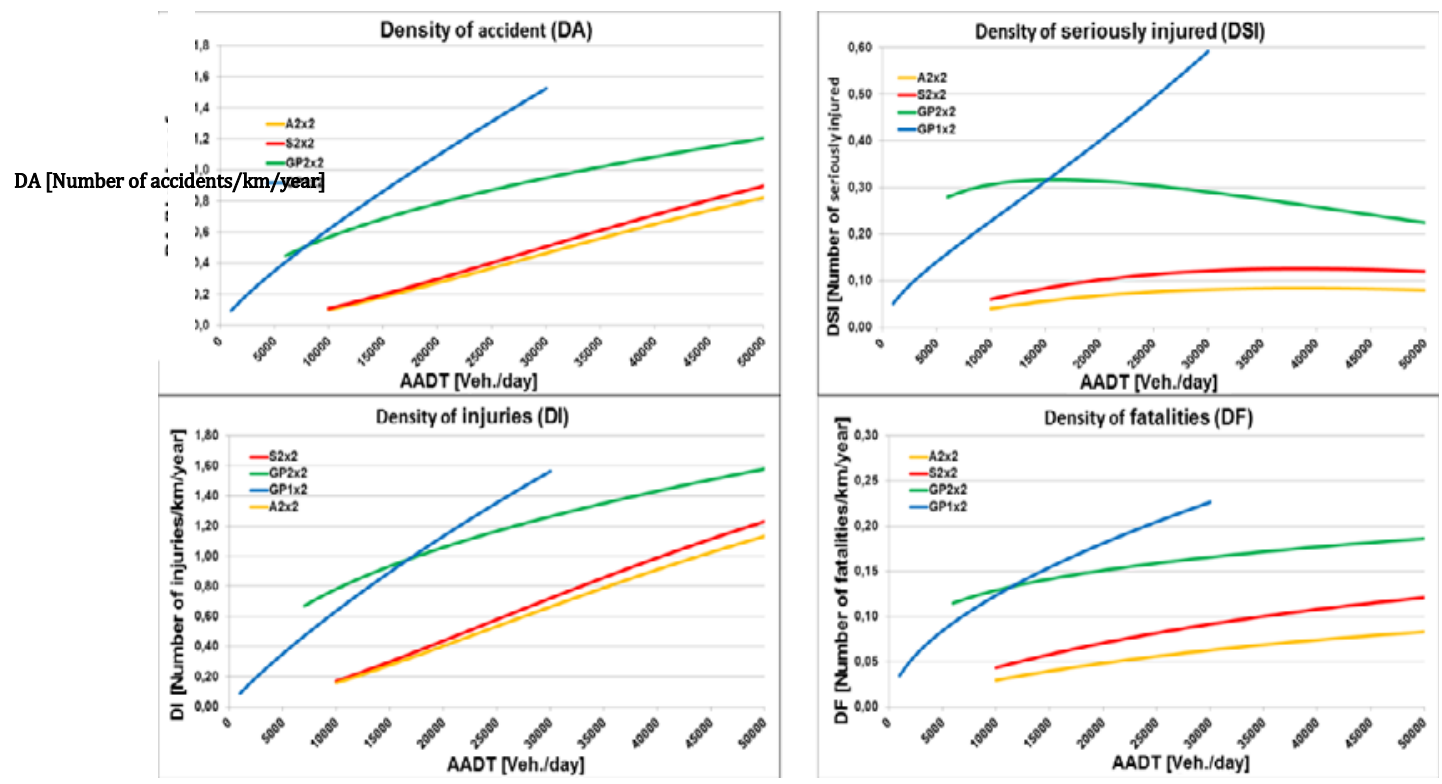

Fig. 1 Road safety measures depending on ADDT intensity.

The authors recommend using the PTV Visum software as one of the elements of the tool Safety PL - Support Tool for Road Safety Impact Assessment. The choice of the PTV Visum software has been dictated by the fact that it is the most commonly used tool for work related to forecasts and analyses of traffic on newly designed roads in Poland. This approach will help to use the results of traffic forecasts for the entire impact area of the planned road, in the prepared models predicting accidents and casualties without the necessity to transfer them to other tools that support the calculation of road safety assessment. The essential element preceding the calculation of road safety measures is preparation of data on homogeneous sections in the impact area. For this purpose, the PTV Visum programme will prepare attributes for all variables in the prepared prediction models of accidents and victims. The modules are shown in Fig. 2. 


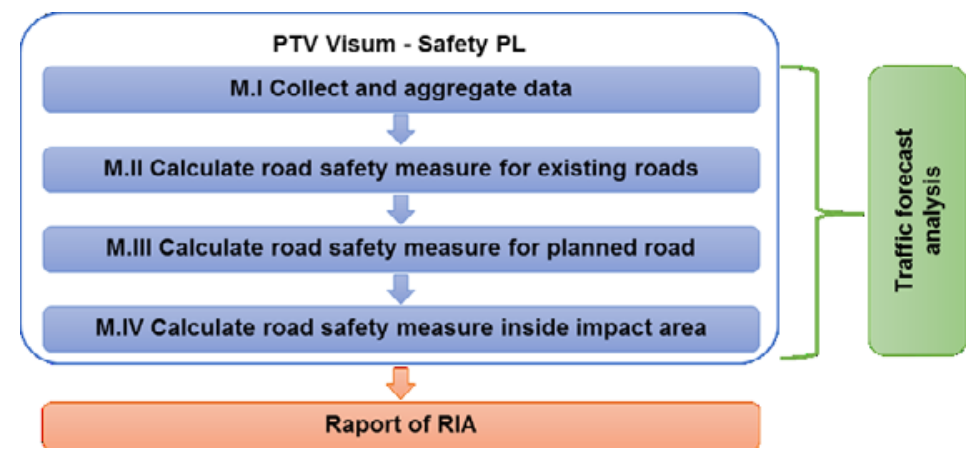

Fig. 2 Safety PL modules.

\section{Road safety audit}

With more than ten years of audit implementation experience in Poland [7] two groups of problems can be identified. They are related to:

- the process of road design and use of safety standards,

- the prevalence, correctness and effectiveness of the auditing procedures.

In the first group of problems, road safety audit shows that statistically the same errors keep appearing quite often when it comes to designing the cross-section and vertical alignment, layout, junctions and interchanges:

- the use of $1 \times 4$ and 1x6 cross-sections with no central reservation,

- structures (utility poles, barriers) are placed on narrow pavements (1.5-2.0 m),

- cyclists and pedestrians are not effectively segregated in the street cross-section,

- steep slopes are used in hazardous places,

- sight distance is not sufficient on horizontal and vertical curves,

- the distances between junctions are too small; junctions are classified in the design as exits

- poor surface drainage of the carriageway,

- selection of the wrong junction type,

- interchanges not matching traffic parameters.

The second group of problems arise due to difficulties with ensuring:

- professional staff and independence of auditors' comments,

- objectivity in assessing a design's proposals for their safety and reasonable recommendations.

At present, there are three documents that are related directly to audit procedures:

- Regulation 42 of the Director General for National Roads and Motorways of 3 September 2009 concerning the road safety impact assessment and road safety audits of road infrastructure projects,

- Act of 13 April 2012 revising the public roads act and some other acts introducing the road safety audit - an independent, detailed and technical assessment of a public road being designed, built, improved or used for the safety of road users,

- Ordinance of the Minister of Transport, Construction and Maritime Economy of 14 September 2012 concerning training and certificates for road safety auditors.

\section{Managing the safety of an existing road network}

The main objective of road safety ranking (RSM) is to select sections that carry the highest individual risk, i.e. the likelihood of being involved in fatal crash of a road user and sections 
that carry the highest societal risk and the biggest potential for reducing accident costs as a result of road authority actions [8]. The intermediate goals of RSM are to:

- systematically assess safety on existing road networks,

- identify and rank high risk sections,

- identify and rank sections with the highest density of accident costs and sections with the highest potential to reduce accident costs,

- create a basis for selecting sections that need work of the highest effectiveness.

In the traffic safety ranking five classes are proposed depending on the potential for reducing accident costs on road sections (A, B, C, D, E) [9]. While hazardous sections must be ranked on national roads only, in 2015 a new ranking was developed for the National Road Safety Council covering regional roads [10]. Fig. 3 shows an example of the ranking looking at societal risk (density of accident costs) for run-off-road accidents.

For the particular technical classes of national roads the risk of an accident was assigned at three levels of acceptance (Table 1):

- unacceptable risk level on a road section means a strong likelihood of severe personal or economic consequences - the road section cannot operate safely until that risk is reduced or the sources of the hazard are removed,

- tolerated risk level on a road section means a medium or low likelihood of personal or economic consequences - the road section may operate temporarily or under certain conditions (such as the use of ad hoc solutions to improve safety such as speed limits, a more intensified road traffic enforcement),

- acceptable risk level means low or very low likelihood of personal or economic consequences - the road section can operate with no additional measures.

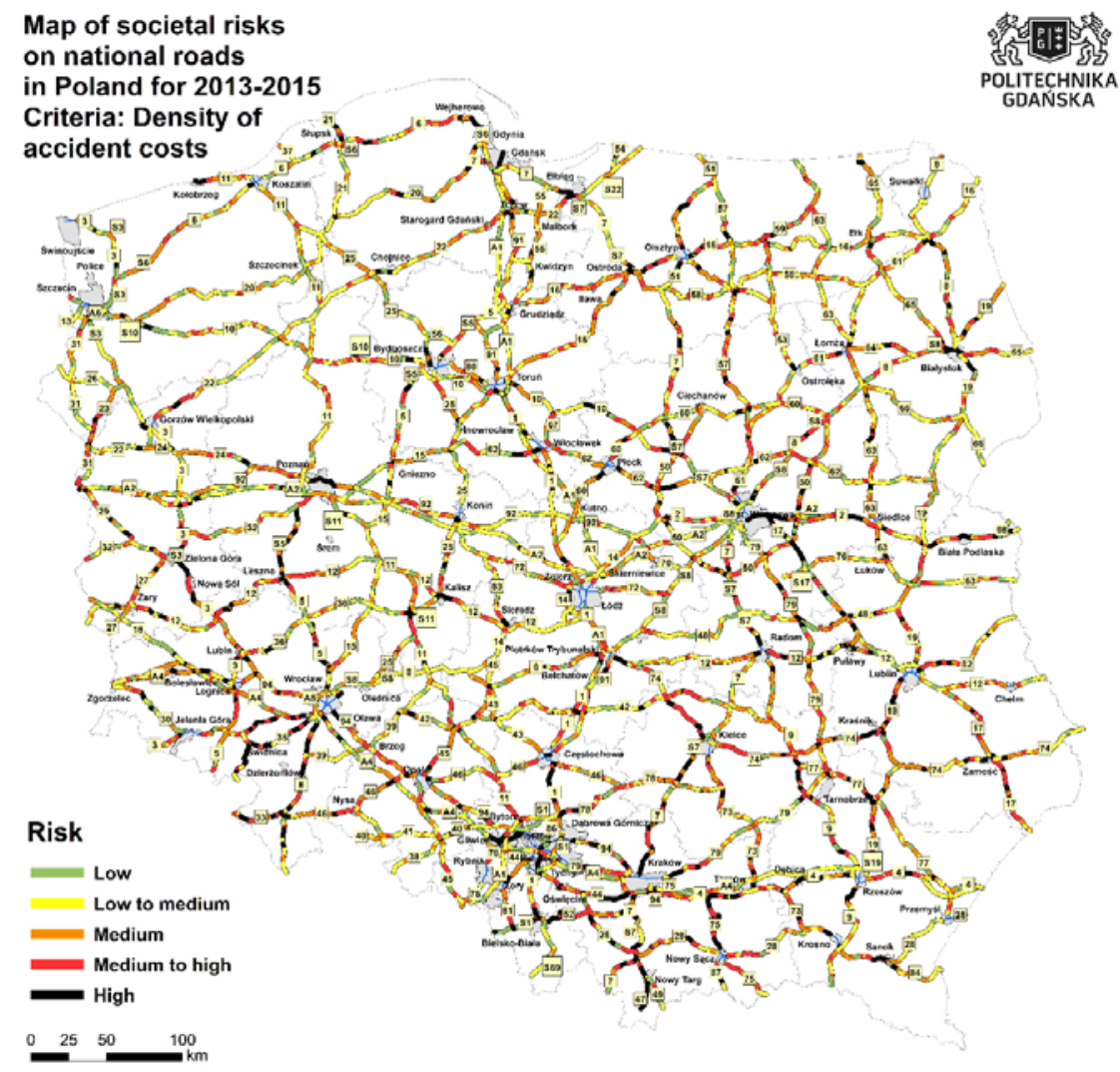

Fig. 3. Map of sections of national roads, societal risk, accident density. 
Tab.1 Requirements for risk management on the network of national roads.

\begin{tabular}{|c|c|c|c|}
\hline \multirow{3}{*}{ Road technical class } & \multicolumn{3}{|c|}{ Level of acceptance of fatality accident risk } \\
\hline & Acceptable risk & Tolerated risk & Not tolerated risk \\
\hline & \multicolumn{3}{|c|}{ Classes of risk on a road section } \\
\hline Motorway & A & B, C & D, E \\
\hline Express road & A & B, C & $\mathrm{D}, \mathrm{E}$ \\
\hline Main road of fast traffic & A, B & C, D & $\mathbf{E}$ \\
\hline Main road & A, B & C, D & $\mathbf{E}$ \\
\hline
\end{tabular}

\section{Road safety inspection}

Three types of inspection are distinguished: general (IO), detailed (ID) and special (IS). General inspections are conducted cyclically. Detailed and special inspections are conducted on road sections that have an E level of risk or as needed [8]. The road safety inspection applies to all road objects and phenomena that are important for road safety which occur on roads and in the clear zone, especially in the area of geometry, traffic layout, surrounding, visibility and road and roadside parameters in relation to the required speed (Fig. 3 - long pedestrian crossing, wrong solutions of the road intersection, trees, trees at the roadside, poor visibility).
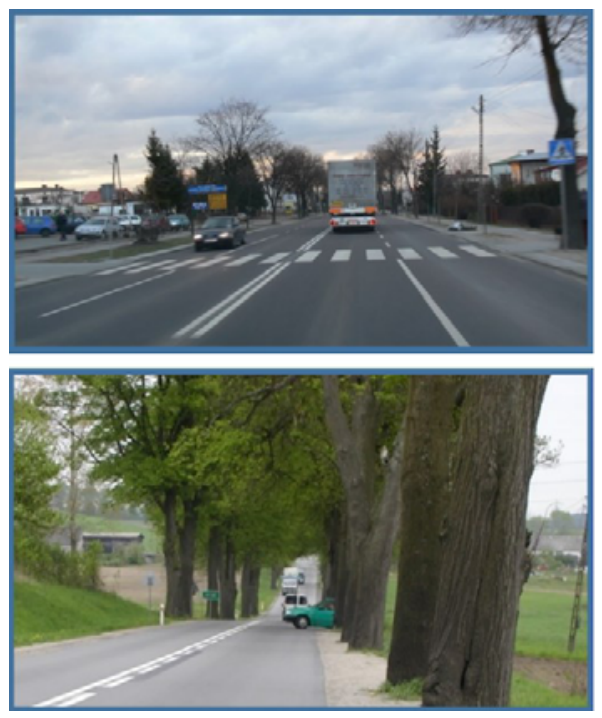
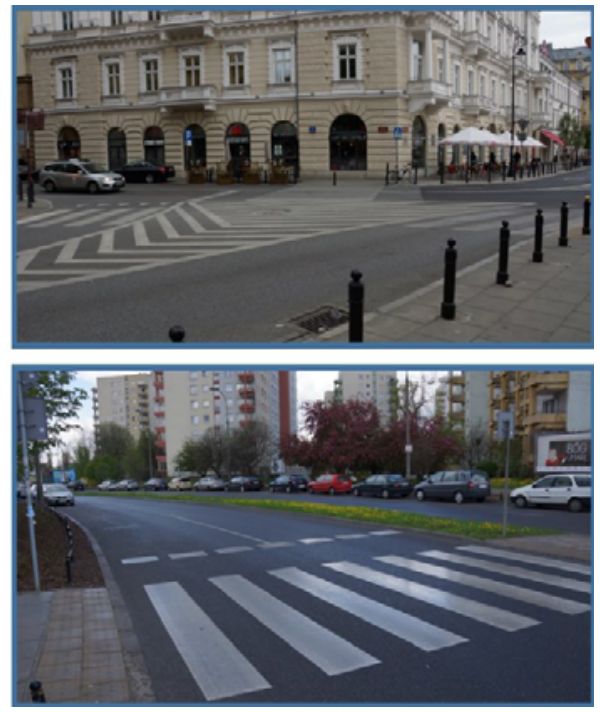

Fig. 3 Examples of road defects.

Once the hazard assessment is completed, the next step is to determine whether the hazard will be removed or reduced to reduce the potential risk or whether hazard protection measures will be introduced. Based on this, steps should be taken to solve the problem. After a period of verification of the method (2 years from implementation), three classes of defects have been adopted ( $\mathrm{A}$ - small risk, $\mathrm{B}$ - medium risk, $\mathrm{C}$ - high risk). At present, work is ongoing on objective road risk measures. The effects of speed and traffic volume on risk class must be defined. An outcome of the research will be a tool to help with objective ranking of road and roadside hazards identified during inspections. 


\section{Summary}

The tools for road infrastructure safety management presented above need more research and a broader scope of application. If we can develop models of how selected factors impact road safety measures (to ensure a better selection of remedial measures), prepare road safety management methods at the operational level and introduce the Directive [2] to the network of regional roads, road safety in Poland can continue to improve.

\section{References}

1. K. Jamroz, Method of risk management in highway engineering. Metoda zarządzania ryzykiem w inżynierii drogowej (Gdansk University of Technology, Gdansk, 2011)

2. Directive of the European Parliament and of the Council of 19 November 2008 on road infrastructure safety management 2008/96/EC (Official Journal of the European Union L.319/59) (2008)

3. K. Jamroz, M. Budzyński, W. Kustra, L. Michalski, S. Gaca, Tools for road infrastructure safety management - Polish experiences, in: Transp. Res. Procedia, pp. 730-739 (2014). doi:10.1016/j.trpro.2014.10.052

4. W. Kustra, K. Jamroz, M. Budzyński, Safety PL - a support tool for Road Safety Impact Assessment, in: 6th Transp. Res. Arena, (Elsevier, 2016)

5. K. Jamroz, The impact of road network structure and mobility on the national traffic fatality rate, Procedia - Soc. Behav. Sci. 54 pp. 1370-1377 (2012). doi:10.1016/j.sbspro.2012.09.851

6. T. Szczuraszek, J. Kempa, G. Bebyn, J. Chmielewski, et al., GAMBIT Kujawsko Pomorski, Tom II. Road safety improvement program (Diamond Books, Bydgoszcz, 2012)

7. K. Jamroz, S. Gaca, W. Kustra, L. Michalski, Road Safety Auditor's Guide - Part I Road Traffic Safety Impact Assessment of Road Infrastructure Projects, Part II Road Safety Audit - Prepared for GDDKiA (Cracow University of Technology, Gdansk University of Technology, 2011)

8. M. Budzyński, K. Jamroz, W. Kustra, S. Gaca, L. Michalski, Classification of dangerous road sections on national roads - Prepared for GDDKiA (Gdansk University of Technology, Cracow University of Technology, 2013)

9. EuroRAP, Atlas of Risks on National Roads in Poland in 2009-2011, (n.d.)

10. K. Jamroz, W. Kustra, J. Wachnicka, M. Berkowski, Risk classification methodology for selected types of road accidents on provincial roads as well as for voivodships and poviats (Ministry of Infrastructure and Development, 2015) 